\title{
Experimental evaluation of peer-to-peer applications
}

\author{
Roberto Canonico · Claudia Canali · Walid Dabbous
}

Received: 10 October 2012 / Accepted: 16 October 2012 / Published online: 8 November 2012

(C) Springer Science+Business Media New York 2012

Peer-to-peer applications produce nowadays a significant fraction of the whole Internet traffic. The peerto-peer paradigm is not only used for file sharing and content dissemination but also for live video broadcasting, multiparty interactive gaming, as well as for large scale VoIP and teleconferencing applications. Existing peer-to-peer applications and protocols have been designed for peers running in normal PCs equipped with a broadband connection to the Internet. However, the emerging scenario is much more complex and heterogeneous, including a variety of terminals (PDAs, NAS boxes, etc.) and access networks (xDSL, Wi-Fi, WiMax, Wireless Mesh Networks, etc.).

Simulation has been the most widely used approach for performance evaluation of peer-to-peer applications because it represents a fast and inexpensive way to test novel solutions. Nonetheless, the growing complexity and heterogeneity of the emerging scenario for peerto-peer applications has made it necessary to validate models and simulation results through experimental evaluation in realistic settings. This need is testified by the recent trend of conducting evaluation of peer-topeer applications in large scale testbeds (e.g. Planet$\mathrm{Lab}$ ) as well as in the real world (e.g. by collecting data

R. Canonico $(\varangle)$

Università degli Studi di Napoli Federico II, Napoli, Italy

e-mail: roberto.canonico@unina.it

C. Canali

University of Modena and Reggio Emilia, Modena, Italy

e-mail: claudia.canali@unimore.it

W. Dabbous

INRIA, Sophia Antipolis, France

e-mail: walid.dabbous@inria.fr from existing BitTorrent trackers or by participating in real peer-to-peer networks with instrumented peers). Performing an experimental evaluation of distributed applications on a large scale, however, is still a challenging task. Firstly, it requires resources which should be made available to the community of researchers through a cooperative effort of institutions. Secondly, but not least important, it should be conducted with a sound methodology and produce results that could be analysed and reused by the whole community of researchers.

Our call for papers explicitly asked for contributions illustrating either experiences in conducting real-world experimental analysis of peer-to-peer applications or innovative methodologies that could be useful for assessing such applications with respect to non-functional requirements, such as performance, scalability, fault tolerance, availability and security. The call for papers attracted 13 submissions. Each paper was carefully reviewed by at least three reviewers. At the end of the reviewing process, we selected six papers that we present in this special issue.

The first three papers present how to setup and manage large scale experimental scenarios.

"Characterization of Community Based-P2P Systems and Implications for Traffic Localization" by Ruben Torres et al. presents a characterization of two different closed community-based P2P applications in terms of both application parameters as well as network traffic. This study also investigates the relation between application behaviour and network topology, providing useful insights for traffic optimization strategies in ISP's networks.

In "Experiences with large-scale operational trials of ALTO-enhanced P2P Filesharing in an intra-ISP 
scenario", Mischa Schmidt et al. evaluate the effectiveness of an application-level traffic optimization strategy for a peer-to-peer filesharing application by illustrating an extensive intra-ISP measurement campaign conducted in a real operator network.

"Detection and mitigation of localized attacks in a widely deployed P2P network" by Thibault Cholez et al. presents the results of a large scale monitoring campaign aimed at detecting malicious peer insertions in KAD, a widely deployed DHT-based peer-to-peer network. This paper also presents a countermeasure against this kind of attacks. The efficiency of the proposed protection scheme was experimentally evaluated in PlanetLab.

The next two papers propose emulative approaches to evaluate the performance of real peer-to-peer networks.

"Validation of H-P2PSIP, a scalable solution for interoperability among different overlay networks" by Isaias Martinez-Yelmo et al. investigates the scalability properties of H-P2P SIP, a hierarchical overlay architecture for interoperability of different peer-topeer networks. The authors present in details how they setup a complex experimental scenario comprising 1000 real peers by combining virtual machines and emulated networks.

In "ModelNet-TE: An emulation tool for the study of P2P and Traffic Engineering interaction dynamics", Dario Rossi et al. present a tool that allows to study the mutual effects of an ISP's Traffic Engineering practices and real peer-to-peer applications. The effectiveness of the tool is evaluated by investigating the effect of a minimum congestion load balancing policy on two applications (BitTorrent and WineStreamer) in realistic topologies.

Finally, Marco Biazzini and Alberto Montresor present in "P2POEM: Function Optimization in P2P Networks" an innovative framework that aims at easing the burden of performing function optimization tasks in a decentralized P2P network of solvers. The authors also evaluate the effectiveness of the proposed approach by experimentally evaluating a distributed Differential Evolution method for the optimization of multidimensional functions executed in a peer-to-peer network of computing nodes.

Acknowledgements The editors would like to thank all authors who submitted their work to this special issue. Last but not least we would also like to express our sincere gratitude to the following colleagues who kindly collaborated with us during the review process.

- Al-Mukaddim Khan Pathan

- Alberto Montresor
- Alessio Botta

- Andreas Mauthe

- Arnaud Legout

- Carmen Guerrero

- Chadi Barakat

- Dali Kaafar

- Dario Rossi

- Giovanni Di Stasi

- Giovanni Stea

- Laurent Schumacher

- Luca Vollero

- Maria Luisa Merani

- Michele Amoretti

- Mohamed Ali Kaafar

- Paolo Veglia

- Pietro Michiardi

- Riccardo Lancellotti

- Roberto Bifulco

- Ruben Cuevas Rumin

- Simon Pietro Romano

- Stefano Avallone

- Stevens LeBlond

- Subhabrata Sen

- Thierry Rakotoarivelo

- Thierry Turletti

- Vittorio Scarano

- Yong Cui

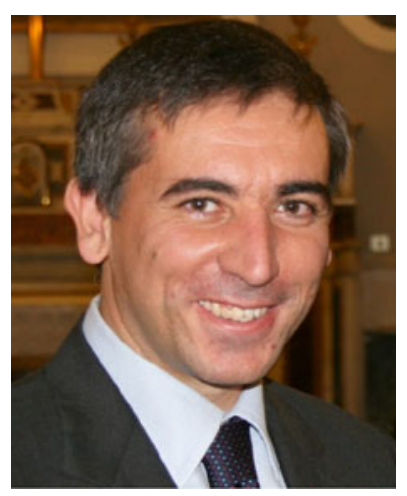

Roberto Canonico is associate professor at University of Napoli Federico II. He received the Laurea degree (cum laude) in electronic engineering from University of Napoli Federico II in 1995, and a Ph.D. in computer engineering from the same University in 2000. In 2000, he was a visiting member of the Distributed Multimedia Research Group at Lancaster University, UK. Roberto has been involved in several European Research Projects, such as CADENUS, INTERMON, ONELAB and ONELAB2, and Networks of Excellence, such as E-NEXT and CONTENT. His current research interests include experimental research infrastructures and testbeds, network virtualization, cloud computing, peer-to-peer traffic optimization, overlay networks, network emulation and simulation. 


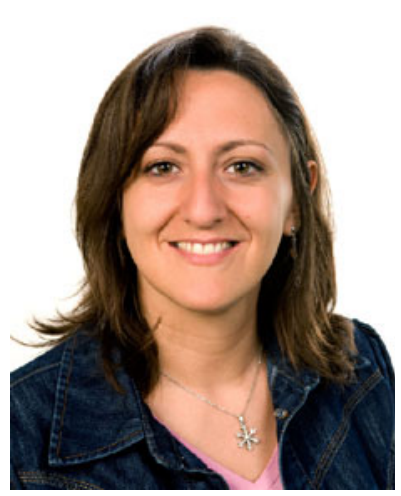

Claudia Canali is a researcher at the University of Modena and Reggio Emilia since 2008. She received the Laurea degree summa cum laude in computer engineering from the same university in 2002, and the Ph.D. degree in information technologies from the University of Parma in 2006. During 2004 she spent eight months as visiting researcher at the AT\&T Research Labs in Florham Park, New Jersey. Her research interests include high performance architectures for Internet-based services, cloud computing, peer-to-peer systems and wireless networks for mobile Web access.

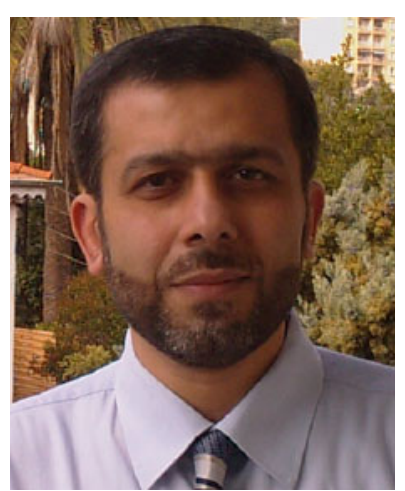

Walid Dabbous is a senior researcher at INRIA where he leads the Planete research team on networking. He received his DEA and his Doctorat d'Universite from the University of Paris XI in 1987 and 1991 respectively. He participated to several FP7 research projects such as: Muse, E-NEXT, OneLab and OpenLab. He is also involved in the French Equipment of Excellence platform project FIT (Future Internet of the Things). His current research interests include: Information Centric Networking Protocols, Networking Experimental Platforms and Simulators and $\mathrm{P} 2 \mathrm{P}$ systems performance and privacy. 